\title{
Scanning the journals
}

\begin{abstract}
Ultrasound and autism association, link or coincidence?
Abramowicz J

Journal of Ultrasound in Medicine 2012; 31: 1261-1269

This review article is timely. Autism was wrongly linked to vaccination by an unscrupulous and now discredited clinician. So as the autism spectrum disorders seem to be on the rise and so is obstetric ultrasound usage, it is not surprising that someone somewhere will attempt to correlate the two.

Abramowicz sifts through the evidence and, as expected from a leader in the field of ultrasound safety, is meticulous in his evaluation. He concludes that there is no evidence that a cause-effect relationship exists between in utero clinical ultrasound exposure and autism. However, he rightly points out that, as ultrasound has thermal and mechanical effects on tissue, its use should be restricted to medical indications, by trained professionals, for as short a period and as low an intensity as compatible with accurate diagnosis.
\end{abstract}

\section{Fetal CNS scanning - less of a headache than you think Monteagudo A and Timor-Trisch IE Clinical Obstetrics and Gynecology 2012; 55 (1): 249-265}

These authors are experts in the 'fetal neuroscan' as they call it. I would recommend this paper as it details the normal anatomy of the fetal brain and the basic scan as well as what constitutes the

While much of what they demonstrate will be known to us all, the multiplanar 3D fetal neuroscan may be less familiar. Using their systematic approach, however, should make CNS fetal scanning less arduous.

As they conclude, ordering an MRI as a first line diagnostic test when the question of a CNS anomaly arises, is tempting but usually unnecessary.

\section{Contingent screening for Down syndrome completed in the first trimester: a multicenter study}

Muñoz-Cortes M, Arigita M, Falguera G, Seres A, Guix D, Baldrich E, et al.

Ultrasound Obstetrics and Gynecology 2012; 39: 396-400

This group of 14 authors from Catalonia, Spain, assessed prospectively the feasibility and effectiveness of a first trimester contingent strategy for Down syndrome detection in a large cohort of pregnant women in their region. They used Nicolaides, et al's proposal of a combined test (NT, serum PAPPA, free betaHCG) as a first stage screen. Then women in the intermediate risk category (i.e. a Down syndrome risk of between 1:100 to 1:1000) had the second step - nasal bone, ductus venosus and tricuspid flows assessment. The aim of course is to reduce the false positive rate without affecting the Down syndrome detection rate.

They found that they did indeed reduce the false positive rate from $3.0 \%$ to $1.7-1.8 \%$ with the contingency strategy. The detection rates were similar between combined testing and category screening. However they discovered two main drawbacks, which I suspect we might find here if and when we adopt the contingency approach: the women at high intermediate fetal neuroscan and when to consider it.

risk still preferred invasive testing and rebooking patients for the second scan before 14 weeks gestation for women with low intermediate risk was "a logistical challenge". In other words patients voted with their feet. This "real world" study is the first prospective one of its kind evaluating contingency screening and is a valuable lesson for those who would apply its theoretical benefits to all.

\section{Ultrasound-guided interscalene blocks \\ Garlin A and Warren L Journal of Ultrasound in Medicine 2012; 31: 979-983}

In this invited article the authors remind us of the evolution of the use of ultrasound by anaesthetists for peripheral nerve blocks. Instead of using the traditional anatomical landmarks technique, they advocate ultrasound guidance for local anaesthesia prior to shoulder surgery. Their review demonstrates that ultrasound guidance of needle placement is efficient and decreases the time needed for satisfactory interscalene blocks. Safety issues have been less well studied of reduction in the amount of anaesthetic needed and there is evidence in the incidence of vascular punctures.

There has been increased use of regional anaesthesia in other areas mainly due to the adoption of ultrasound in needle-placement. Ultimately the patient benefits as a general anaesthetic can be safely avoided.

The competency-based mandate for emergency bedside sonography training and a tale of two residency programs Jang TB, Coates WC and Lui YI Journal of Ultrasound in Medicine 2012; 31: 515-521

Anyone interested in training sonographers might want to read this article from the UCLA and George Washington Schools of Medicine. I found it contained much food for thought. Medical educators know the difference between competence and competency.

Competence in emergency sonography would be the ability to get the right images. Competency requires the emergency physician to integrate the ultrasound findings into safe patient management in the emergency room.

The authors discuss the different training approaches for resident doctors, each with educational goals, learning objectives and interim assessments. No one yet knows the best approach and the authors are interested in data from other programs.

Simulation training is not addressed but, while expensive, it might protect patients.

ASUM's main role is education and maintenance of standards across the ultrasound spectrum. This article may stimulate discussion amongst trainers.

\section{The Gleaner}

